\title{
Concurrent image-guided intensity modulated radiotherapy and chemotherapy following neoadjuvant chemotherapy for locally advanced nasopharyngeal carcinoma
}

Pei-Wei Shueng ${ }^{1,4}$, Bing-Jie Shen', Le-Jung Wu', Li-Jen Liao ${ }^{2}$, Chi-Huang Hsiao ${ }^{3}$, Yu-Chin Lin ${ }^{3}$, Po-Wen Cheng ${ }^{2}$, Wu-Chia Lo ${ }^{2}$ Yee-Min Jen ${ }^{4}$ and Chen-Hsi Hsieh ${ }^{1,5^{*}}$

\begin{abstract}
Background: To evaluate the experience of induction chemotherapy followed by concurrent chemoradiationwith helical tomotherapy (HT) for nasopharyngeal carcinoma (NPC).

Methods: Between August 2006 and December 2009, 28 patients with pathological proven nonmetastatic NPC were enrolled. All patients were staged as IIB-IVB. Patients were first treated with 2 to 3 cycles of induction chemotherapy with EP-HDFL (Epirubicin, Cisplatin, 5-FU, and Leucovorin). After induction chemotherapy, weekly based PFL was administered concurrent with HT. Radiation consisted of 70 Gy to the planning target volumes of the primary tumor plus any positive nodal disease using 2 Gy per fraction.

Results: After completion of induction chemotherapy, the response rates for primary and nodal disease were 96.4\% and 80.8\%, respectively. With a median follow-up after 33 months (Range, 13-53 months), there have been 2 primary and 1 nodal relapse after completion of radiotherapy. The estimated 3-year progression-free rates for local, regional, locoregional and distant metastasis survival rate were $92.4 \%, 95.7 \%, 88.4 \%$, and $78.0 \%$, respectively. The estimated 3-year overall survival was $83.5 \%$. Acute grade 3,4 toxicities for xerostomia and dermatitis were only $3.6 \%$ and $10.7 \%$, respectively.

Conclusion: HT for locoregionally advanced NPC is feasible and effective in regard to locoregional control with high compliance, even after neoadjuvant chemotherapy. None of out-field or marginal failure noted in the current study confirms the potential benefits of treating NPC patients by image-guided radiation modality. A long-term follow-up study is needed to confirm these preliminary findings.
\end{abstract}

Keywords: Concurrent chemoradiation, Intensity-modulated radiotherapy, Helical tomotherapy, Nasopharyngeal carcinoma

\section{Background}

Locally advanced NPC patients present with poor prognosis. This has led to increasing interest in exploring the use of chemotherapy. Recently, meta-analysis has confirmed the superiority of concurrent chemoradiation (CCRT) over radiotherapy (RT) alone in terms of survival or locoregional control among patients with locally

\footnotetext{
* Correspondence: chenci28@ms49.hinet.net

'Division of Radiation Oncology, Department of Radiology, Far Eastern Memorial Hospital, Taipei, Taiwan

Full list of author information is available at the end of the article
}

advanced NPC [1-3]. However, the optimal regimen and scheduling remains to be determined and efforts to improve the increased toxicities are still unremitting.

With the improvement of RT techniques, such as intensity-modulated radiotherapy (IMRT) or imageguided radiotherapy (IGRT), radiation oncologists have the ability to deliver tumoricidal doses to the target while maintaining tolerable doses to critical organs. Recently, several non-randomized studies have demonstrated impressive tumor control and survival using IMRT in NPC. Moreover, the predominant failure

\section{C) Biomed Central}


pattern is now distant failure rather than local failure [4]. To conquer distant metastasis, adding induction chemotherapy or adjuvant chemotherapy to concurrent chemoradiation is still an attractive approach that needs to be clarified.

Helical tomotherapy (HT), an innovative image-guided IMRT device, can perform daily CT image registration before treatment and deliver 51-angled rotational IMRT. Our institute started the first HT treatment using Tomotherapy Hi-Art systems (Tomotherapy, Madison, WI) in December 2006. Using HT, we have previously reported encouraging experiences for oropharyngeal [5], postoperative treatment of high-risk oral cavity cancer [6] and cervical cancer [7]. In comparison with conventional IMRT, the HT results have demonstrated better dosimetry coverage and highly conformal dose distributions to the targets and the impressive ability to simultaneously spare critical organs. In the treatment of nasopharyngeal carcinoma, tomotherapy plans were superior to IMRT plans in conformity and homogeneity of planning target volume (PTV) and the sparing of the critical organs at risk (OARs) [8].

We herein report our preliminary experience of concurrent helical tomotherapy plus chemotherapy following induction chemotherapy for locally advanced NPC, with special focus on response rate, acute treatmentrelated sequelae and failure pattern and locoregional control.

\section{Methods}

\section{Patient Characteristics}

Between August 2006 and December 2009, 28 patients with pathological proven NPC were enrolled in this retrospective analysis. All of the patients were diagnosed as non-metastatic NPC in the cancer work-up initially. Approval for the study was obtained from the Institutional Review Board of Far Eastern Memorial Hospital (FEMH No. 100050-E). The clinical characteristics are detailed in Table 1. There were 22 men and 6 women with a median age of 47.5 years. Most patients (85.7\%) had pathology of WHO type III (undifferentiated carcinoma). Patients were staged according to the 2002 American Joint Committee on Cancer (AJCC) staging system. All patients were staged as having locally advanced disease (stage IIB-IVB). Table 2 detailed the TNM distribution of the patients.

Staging workups included complete histories and physical examinations, fiberoptic endoscopic evaluation, complete blood counts, liver and renal function tests, chest X-rays, abdominal ultrasound, magnetic resonance imaging (MRI) scans of the head and neck region, bone scan and dental evaluation. CT scans of the chest and abdomen were obtained whenever possible before the beginning of treatment if distant metastasis was
Table 1 Characteristics of 28 patients

\begin{tabular}{lll}
\hline Variable & number & percent \\
\hline Gender & 22 & $78.6 \%$ \\
$\quad$ Male & 6 & $21.4 \%$ \\
$\quad$ Female & & \\
Stage (AJCC, 2002) & 3 & $10.7 \%$ \\
IIB & 15 & $53.6 \%$ \\
III & 10 & $35.7 \%$ \\
IVA/B & & \\
T stage & 11 & $39.3 \%$ \\
T1-T2 & 17 & $60.7 \%$ \\
T3-T4 & & \\
N stage* & 8 & $28.6 \%$ \\
NO-N1 & 20 & $71.4 \%$ \\
N2-N3 & & \\
Field-dose arrangement & 24 & $85.7 \%$ \\
SIB & 4 & $14.3 \%$ \\
Conventional shrinking field & & $14.3 \%$ \\
Pathology & 4 & $85.7 \%$ \\
WHO I \& II & 24 & \\
WHO III & &
\end{tabular}

suspected by abnormal finding in chest $\mathrm{x}$-ray or abdominal ultrasound.

\section{Chemotherapy}

All patients were treated with induction chemotherapy followed by CCRT with HT. Induction chemotherapy regimens, EP-HDFL, consisted of Epirubicin $40 \mathrm{mg} / \mathrm{m}^{2}$, 30 minutes infusion, followed by Cisplatin $60 \mathrm{mg} / \mathrm{m}^{2}, 5$ FU $2000 \mathrm{mg} / \mathrm{m}^{2}$, and Leucovorin $300 \mathrm{mg} / \mathrm{m}^{2}$, 24 hours infusion on day 1 , and 5 -FU $2000 \mathrm{mg} / \mathrm{m}^{2}$, and Leucovorin $300 \mathrm{mg} / \mathrm{m}^{2}, 24$ hours infusion on day 8 and 15 , repeated every 4 weeks. Three cycles were planned unless severe side effects occurred. Chemotherapy during the CCRT phase, PFL, consisted of Cisplatin $30 \mathrm{mg} /$ $\mathrm{m}^{2}$, 5-FU $450 \mathrm{mg} / \mathrm{m}^{2}$ as bolus, and Leucovorin $30 \mathrm{mg} /$ $\mathrm{m}^{2}$, on a weekly basis. Curative radiotherapy began within 3 weeks after completion of the last cycle of induction chemotherapy.

Table 2 Dose-volumetric statistics for target volumes

\begin{tabular}{lcc}
\hline Parameters & \multicolumn{2}{c}{ Mean (range) } \\
\cline { 2 - 3 } & PTV $_{\mathbf{7 0}}$ & PTV $_{\mathbf{6 3}}$ \\
\hline Volume (cc) & $253.8(61.7-776.1)$ & $528.8(175.8-1213.2)$ \\
Mean dose (Gy) & $71.9(70.1-75.3)$ & $64.3(54.2-68.8)$ \\
Maximum dose (Gy) & $74.4(70.3-79.7)$ & $69.4(54.6-76.1)$ \\
Minimum dose (Gy) & $60.1(44.9-69.7)$ & $47.4(26.8-57.6)$ \\
D $_{95}(G y)$ & $70.1(68.8-72.0)$ & $61.4(53.9-67.0)$ \\
$V_{97}(\%)$ & $98.3(95.3-100.0)$ & $97.8(94.6-100.0)$ \\
\hline
\end{tabular}




\section{Radiotherapy}

\section{Immobilization and Contouring}

Patients were immobilized using perforated Type-S thermoplastic head frames (MT-CFHN-C; Civco Medical Solutions, Kalona, IA) for head and shoulder immobilization after induction chemotherapy completed. The head frames would be corrected after a significant neck burden reduction during CCRT. A volumetric contrast enhanced CT image in serial $3 \mathrm{~mm}$ slices was acquired for treatment planning.

\section{Target and Normal Tissue Volume Delineation and Constraints}

Target objects and normal structures were outlined slice by slice on the treatment planning CT. On several occasions, RT-planning images were fused with diagnostic MRI to improve target delineation.

The gross tumor volume (GTV) encompassed the gross extent of the primary tumor and involved neck nodes shown by imaging before induction chemotherapy as well as physical examination. Whenever possible, MRI scan done before induction chemotherapy $(24 / 28)$ was used in addition to the CT scan to delineate the GTV with the assistance of a neuroradiologist. A GTV node was outlined to have a nodal size larger than $10 \mathrm{~mm}$ in the short-axis diameter or the presence of central lucency on CT or MRI images. The clinical target volume of 70 Gy (CTV70) included the GTV with an additional $10 \mathrm{~mm}$ margin and GTV of node with an expansion of $5 \mathrm{~mm}$, respectively. The clinical target volume of 63 Gy (CTV63) was designed to include areas at risk for microscopic involvement, as well as the entire nasopharynx, retropharyngeal nodal regions, skull base, clivus, pterygoid fossae, parapharyngeal space, sphenoid sinus, the posterior one third of the nasal cavity/maxillary sinuses that includes the pterygopalatine fossae, and levels I through $\mathrm{V}$ nodal regions. Level II nodes were contoured bilaterally to the base of skull. The clinical target volume of $56 \mathrm{~Gy}$ (CTV56) was designed for the low-risk subclinical disease area. To account for organ motion and patient setup errors, all of the PTV70, PTV63 and PTV56 were defined as CTV plus a margin of $3 \mathrm{~mm}$. For patients treated with the whole-field SIB technique, PTV70, PTV63 and PTV56 were delivered in the same days and all were amenable to be completed in 35 fractions within 7 weeks.

Critical structures included the brainstem, spinal cord, brain, lens, eyeballs, optic chiasma, optic nerve, inner ear, oral cavity, mandible, parotid gland, larynx, and lung. Optimization was performed using the following criteria for dose constraints. The dose constraints for OARs were as follows: (1) brainstem: maximum dose 50 Gy, (2) spinal cord: maximum dose $40 \mathrm{~Gy}$, (3) optic chiasm and optic nerve: maximum dose 45 Gy, (4) mandible: maximum dose 70 Gy or $1 \mathrm{~cm}^{3}$ or less for 70 Gy or more, (5) bilateral parotid glands: mean dose less than $30 \mathrm{~Gy}$, and median dose less than $26 \mathrm{~Gy}$, and whole parotid gland volume, with a dose less than 20 Gy, more than $20 \mathrm{~cm}^{3}$, and (6) middle and inner ear: mean dose less than $50 \mathrm{~Gy}$. The planning OAR volume (PRV) was set as the brain stem and spinal cord with 5$\mathrm{mm}$ margins in the axial plane. The PRVs of the chiasma and optic nerve were set with $3-\mathrm{mm}$ margins in every direction.

\section{Treatment Plan and Delivery}

The field width, pitch, and modulation factor usually used for treatment planning optimization were $2.5 \mathrm{~cm}$, 0.32 , and 3.0, respectively. Maximum importance was given to target dose coverage. The constraints on dose and penalty were adjusted accordingly during optimization. All patients received daily megavoltage CT acquisitions for setup verification.

\section{Follow-up}

The response criteria were as follows: a complete response was defined as complete regression of all evidence of disease; a partial response required a $50 \%$ decrease of the summed products of the two largest perpendicular diameters of all measurable lesions, without an increase in size of more than $25 \%$ in any lesion or the appearance of new lesions; stable disease was defined as no significant change or any change in tumor size that was less than a partial response but not large enough to be considered progressive disease; and progressive disease was defined as an increase of at least $25 \%$ in the size of measurable lesions or the appearance of any new lesion. Response was assessed before the initiation of radiotherapy and 3 months after completion of the treatment, respectively.

The acute toxicity occurring within 90 days since the beginning of RT was assessed weekly throughout the treatment. The toxicities were defined and graded according to the Common Terminology Criteria for Adverse Events, version 3.0 [9].

\section{Statistical methods}

Descriptive statistics (mean, median, and proportions) were calculated to characterize the patient, disease, and treatment features, as well as toxicities after treatment. The OS, PFS, LRPF, and DMF rates were estimated using the Kaplan-Meier product-limit method [10]. Freedom from local progression was defined as the absence of primary tumor upon physical examination and radiographic examination (CT and MRI scan). Durations were calculated from the date of pathologic proof. Differences were considered significant at $\mathrm{p}<$ 0.05. MedCalc statistical software (version 11.2.1.0, MedCalc Software, Mariakerke, Belgium) was used for 
conducting statistical analyses, manipulating data, and generating tables and graphs that summarize data.

\section{Results}

Dose-volume analysis

Dose-volume histograms statistics for the PTV and organs at risk (OARs) are described in table 2 and 3, respectively. The D95 in PTV70 ranged from 68.8 Gy ( $98 \%$ of the prescription dose) to 72 Gy ( $100 \%$ of the prescription dose). The V97 in PTV70 ranged from $97.3 \%$ to $100 \%$. Mean doses to parotid glands were 33.7 Gy (25.90-43.49 Gy) for the right and 34.1 Gy (24.0248.72 Gy) for the left. The other OARs are summarized in Table 3.

\section{Response}

Most patients (85.7\%) were treated with Whole-field SIB (simultaneous-integrated boost) HT techniques. The median follow-up duration was 33 months (range: 13 to 53 months). Thirteen patients received 2 cycles of induction chemotherapy due to severe nausea $(1 / 28)$, neutropenia $(1 / 28)$, sepsis $(1 / 28)$ and partial response with unsatisfactory response judged by medical oncologist (10/28). The remaining underwent 3 cycles of chemotherapy. Primary tumors had a higher response rate to induction chemotherapy (96.4\%) compared with nodal disease $(80.8 \%)$ which was evaluated by endoscopy \& CT or MR for all patients. The complete response rates were $39 \%$ and $27 \%$ for the primary tumor and neck node, respectively. (Table 4) No patients experienced disease progression during chemotherapy. Also, after remission via induction chemotherapy, there were

Table 3 Dose-volumetric statistics for organs at risk (OARs)

\begin{tabular}{lc}
\hline Organs & Mean (range) \\
\hline Spinal cord $\left[D_{\max }(G y)\right]$ & $40.70(29.20-53.84)$ \\
Brainstem $\left[D_{\max }(G y)\right]$ & $50.15(31.49-62.01)$ \\
Right Optic nerve $\left[D_{\max }(G y)\right]$ & $46.08(19.40-76.39)$ \\
Left Optic nerve $\left[D_{\max }(G y)\right]$ & $43.75(7.98-72.70)$ \\
Optic chiasm $\left[D_{\max }(G y)\right]$ & $46.49(25.50-73.06)$ \\
Right inner ear & \\
$\quad D_{\max }(G y)$ & $59.40(47.61-73.53)$ \\
$D_{\operatorname{mean}}(G y)$ & $41.87(24.43-65.37)$ \\
Left inner ear & \\
$\quad D_{\max }(G y)$ & $60.26(40.87-74.55)$ \\
$\quad D_{\text {mean }}(G y)$ & $43.26(23.01-70.41)$ \\
Right parotid gland & \\
$D_{\operatorname{mean}}(G y)$ & $33.71(25.90-43.49)$ \\
$V_{30}$ Gy $(\%)$ & $45.64(29.30-60.00)$ \\
Left parotid gland & \\
$D_{\text {mean }}(G y)$ & $34.09(24.02-48.72)$ \\
$V_{30}$ Gy $(\%)$ & $46.38(27.10-78.39)$ \\
\hline
\end{tabular}

Table 4 Clinical response after induction chemotherapy and 2 months after completion of CCRT

\begin{tabular}{lll}
\hline Response & After Induction & $\begin{array}{l}\text { After } \\
\text { Concomitant } \\
\text { chemotherapy }\end{array}$ \\
No. (\%) & Chemoradiation & \\
\hline $\begin{array}{l}\text { Nasopharynx, by endoscopy \& CT } \\
\text { or MR }\end{array}$ & & \\
SD & $1 / 28(3.6 \%)$ & $0 / 28(0.0 \%)$ \\
PR & $16 / 28(57.1 \%)$ & $2 / 28(7.1 \%)$ \\
CR & $11 / 28(39.3 \%)$ & $26 / 28(92.9 \%)$ \\
Neck node, by CT or MR & & \\
SD & $5 / 26^{*}(19.2 \%)$ & $0 / 26(0.0 \%)$ \\
PR & $14 / 26(53.8 \%)$ & $5 / 26(19.2 \%)$ \\
CR & $7 / 26(26.9 \%)$ & $21 / 26(80.8 \%)$ \\
\hline
\end{tabular}

*Two patients were staged as T4N0, so 26 patients were available for nodal evaluation.

Abbreviations:

SD: stable disease, PR: partial response, CR: complete response.

no patients who had primary or lymph node enlargement during the rest period before CCRT.

After induction chemotherapy, all patients also received CCRT with HT and achieved complete or partial remission either in the primary site or gross neck nodes. The median cycles for patients received chemotherapy during RT were 4 cycles (range: $2-7$ cycles). There were 4 (14.3\%), $2(7.1 \%)$ and $3(10.7 \%)$ of patients received chemotherapy during RT with 5,6 and 7 cycles, respectively. The average weeks for CCRT were $7.8 \pm 1.1$ wks (range: $6-10 \mathrm{wks}$ ). There were 7 (23.3\%) and $2(6.7 \%)$ patients completed the CCRT course within 9 and 10 wks, respectively. The complete response rate of the nodal area (80.8\%) was inferior to primary location (92.9\%). After completion of the whole treatment, small residual tumors were noted either at the primary site or neck with $7.1 \%$ and $19.2 \%$ of patients, respectively. These residual tumors all showed complete regression upon follow-up after 3 months (Table 4).

\section{Treatment outcome}

The estimated 3-year progression-free (PF) rates for local, regional, locoregional and DMF survival rate were $92.4 \%, 95.7 \%, 88.4 \%$, and $78.0 \%$, respectively. The 3-year estimates of locoregional PF for patients with stage II-IV disease were $100 \%, 92.9 \%$, and $76.2 \%$, respectively. The 3 - year estimated OS was $83.5 \%$ (Figure 1). No patient was lost as of follow-up. Three patients and 2 patients died by distant failure and intercurrent disease (one of chemotherapy related septic shock and the other died of cardiac dysfunction probably related to the anthracycline-chemotherapy of cardiac [11]), respectively.

\section{Acute Toxicities}

The median treatment period during CCRT was 54 days (range: 42 to 73 days). No fatal toxicity related to the 


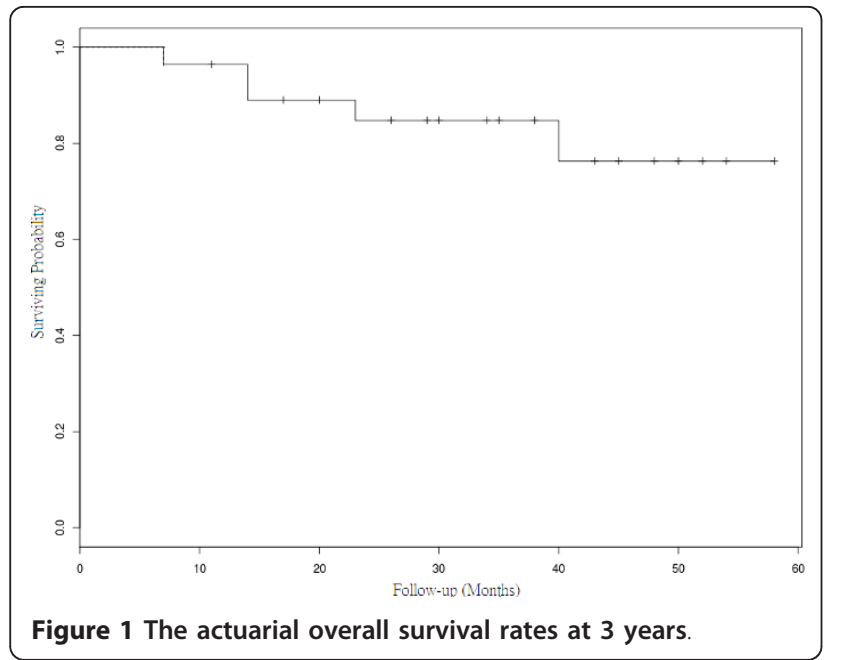

planned treatment occurred in this study. Before induction chemotherapy, all but 3 patients had normal hemogram (Table 5). During induction chemotherapy, grade 3 leukopenia occurred in 1 patient. No patients experienced grade 3 anemia or grade 3 thrombocytopenia. However, the low toxicities of induction chemotherapy are probably due to the low doses of CDDP and Epirubicin.

For CCRT with HT, 4 patients (14.3\%) developed grade 3 leukopenia and 1 patient (3.6\%) developed grade 3 anemia during treatment. Acute nonhematological toxicities related to radiotherapy, including xerostomia and dermatitis, were mostly mild (Table 6.) Only 1 patient had grade 3 xerostomia. Grade 3 or 4 dermatitis was noted in 2 and 1 patients, respectively. However, 13 patients (46.4\%) suffered from grade 3 mucositis. Other grade 3 reactions such as dysphagia and weight loss were noted in 4 and 2 patients, respectively. Seven patients $(25.0 \%)$ needed NG feeding for nutritional supports.

\section{Late Toxicities}

For CCRT with HT, none of patients developed grade 3 toxicities related to radiotherapy, including xerostomia,
Table 6 Acute radiation-related toxicities according to CTCAE v3.0

\begin{tabular}{llllll}
\hline & \multicolumn{5}{c}{ Acute toxicities } \\
\cline { 2 - 6 } Grade & xerostomia & mucositis & dysphagia & dermatitis & weight loss \\
\hline 0 & 0 & 0 & $1(3.60 \%)$ & 0 & $2(7.1 \%)$ \\
1 & $13(46.4 \%)$ & $4(14.3 \%)$ & $8(28.6 \%)$ & $17(60.7 \%)$ & $12(42.9 \%)$ \\
2 & $14(50.0 \%)$ & $11(39.8 \%)$ & $15(53.6 \%)$ & $8(28.6 \%)$ & $12(42.9 \%)$ \\
3 & $1(3.6 \%)$ & $13(46.4 \%)$ & $4(14.3 \%)$ & $2(7.1 \%)$ & $2(7.1 \%)$ \\
4 & 0 & 0 & 0 & $1(3.6 \%)$ & 0 \\
\hline
\end{tabular}

Abbreviations:

CTCAE v3.0: the Common Terminology Criteria for Adverse Events, version 3.0.

dysphagea, dry eyes, trismus and hearing loss. Most of them are normal to grade 1 of toxicities. Only 4/28 patient had grade 2 xerostomia and $1 / 28$ had grade 2 hearing loss.

\section{Failure pattern}

There were $89.3 \%$ (25/28) without locoregional failure. The failure pattern disclosed as follows: local failure only, 2 patients (7.1\%); regional failure only, 1 patient (3.6\%); distant metastases only, 4 patients (14.3\%); and no local plus regional and/or distant failure.

One patient with initial stage IV disease (cT4N1M0) failed locally at the ethmoid sinus 10 months post treatment. After functional endoscopic sinus surgery and adjuvant chemotherapy, the disease was well controlled. (Figure 2A and 2B) Another patient with stage IV disease (cT4N3bM0) failed at the nasopharynx 14 months after the treatment. She then underwent local irradiation plus cetuximab and chemotherapy but died of septic shock. (Figure 2C and 2D)

The only patient who failed for nodal disease with initial stage III disease (cT3N2M0) suffered from left upper neck relapse 16 months after completion of treatment and then was successfully salvaged by neck dissection. (Figure 2E and 2F) No adjuvant treatment was done since only one of 16 dissected nodes showed metastatic lesion. No extracapsular extension or other pathological risk factors were noted. The failure was in a

Table 5 Acute hematological toxicities in $\mathbf{2 8}$ patient after induction chemotherapy and concurrent chemoradiation according to CTCAE v3.0

\begin{tabular}{|c|c|c|c|c|c|c|c|c|c|}
\hline \multirow{2}{*}{$\begin{array}{l}\text { Item } \\
\text { Interval } \\
\end{array}$} & \multicolumn{3}{|c|}{ anemia } & \multicolumn{3}{|c|}{ leucopenia } & \multicolumn{3}{|c|}{ thrombocytopenia } \\
\hline & before & IC & CCRT & before & IC & CCRT & before & IC & CCRT \\
\hline \multicolumn{10}{|l|}{ Grade } \\
\hline 0 & $25(89.3 \%)$ & 11(39.3\%) & $7(25.0 \%)$ & $28(100.0 \%)$ & 19(67.9\%) & $8(29.6 \%)$ & $27(96.4 \%)$ & 17(60.7\%) & 13(46.4\%) \\
\hline 1 & $1(3.6 \%)$ & $13(46.4 \%)$ & $10(35.7 \%)$ & 0 & $5(17.9 \%)$ & $4(14.8 \%)$ & $1(3.6 \%)$ & $11(39.3 \%)$ & $10(35.7 \%)$ \\
\hline 2 & $1(3.6 \%)$ & $4(14.3 \%)$ & 10(35.7\%) & 0 & $3(10.7 \%)$ & $12(44.4 \%)$ & 0 & 0 & $5(17.9 \%)$ \\
\hline 3 & 0 & 0 & $1(3.6 \%)$ & 0 & $1(3.6 \%)$ & $4(11.1 \%)$ & 0 & 0 & 0 \\
\hline 4 & $1(3.6 \%)$ & 0 & 0 & 0 & 0 & 0 & 0 & 0 & 0 \\
\hline
\end{tabular}

Abbreviations:

CTCAE v3.0: the Common Terminology Criteria for Adverse Events, version 3.0. IC: induction chemotherapy (IC); CCRT: concurrent chemoradiation. 

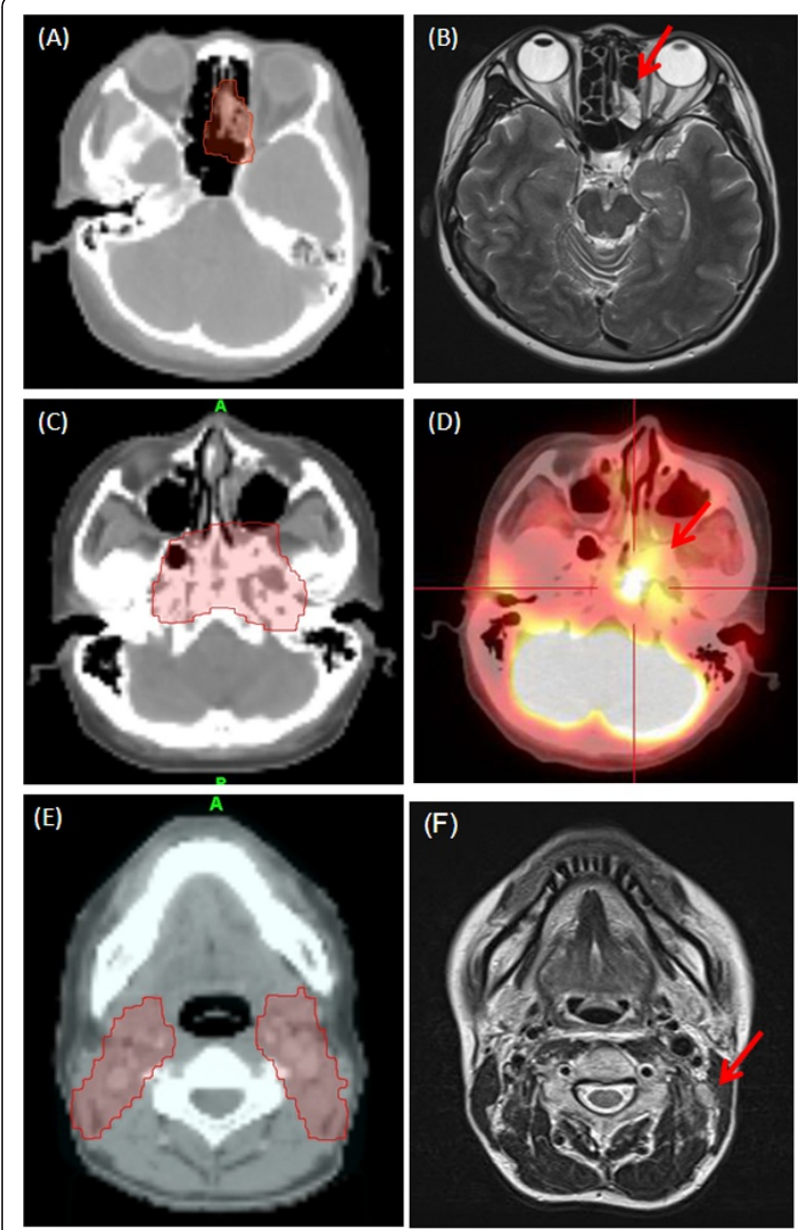

Figure 2 The comparison of original planning dose distribution (red color area) and locoregional failure (red arrow). For patient 1. (A) In 2007/4, planning Dose distribution of $70 \mathrm{~Gy}$ (red color area) at ethmoid sinus; (B) In 2008/1, MRI images show a local relapse. For patient 2. (C) In 2008/11, planning dose distributions of $71.6 \mathrm{~Gy}$ (red color area) at skull base; (D) In 2009/3, PET-CT images show a at skull base, $S U V_{\max }=6.4$. For patient 3. (E) In 2008/7, planning Dose distributions of $70 \mathrm{~Gy}$ (red color area) at neck lymph node and lymphatic drainage; (F) In 2009/6, MRI images show a regional lymph node relapse.

previously irradiated field. No patients failed at the field margins or out of RT fields. The local and regional controls after salvage treatment were $96.4 \%$ and $100 \%$, respectively.

Four patients developed distant metastases over the bone, liver, liver plus bone, and lung at the 6th, 7 th, 10th and 40th month after completion of treatment. All of these 4 patients had N2 disease (stage III and IV). The average relapse time was 8 months. Three of them were died of disease progression and only one patient with liver metastases is still alive with disease and now under systemic treatment. We observed no parotid or dermal failure.

\section{Discussion}

Impressive clinical data of NPC treated by IMRT have been reported in recent years. In one study, the 4-year local progression-free and regional progression-free rates for loco-regional advanced NPC patients were $97 \%$ and $98 \%$, respectively [12]. Recent results from Hong Kong and the Memorial Sloan-Kettering cancer center have also shown similar findings [13-15]. However, with integration of aggressive concurrent chemoradiotherapy schedules, the changing failure pattern has been noted in several publications $[12,16,17]$ and the distant metastases rates, nevertheless, can be as high as $30 \%$ [4].

To conquer the problem of distant metastases, adding neoadjuvant chemotherapy or adjuvant chemotherapy with concurrent chemoradiation is still an attractive approach that needs to be clarified, although post experience is very sparse. A study conducted in Hong Kong [18] reported that 24/25 locally advanced NPC patients achieved partial remissions after induction chemotherapy. Additionally, the 3-year local-PF, regional$\mathrm{PF}$, and DM-PF survival rates were $89.6 \%, 87.2 \%$, and $80.4 \%$, respectively. China has report the largest series of concurrent chemotherapy and IMRT data, with 323 locoregionally advanced NPC patients with neoadjuvant or adjuvant chemotherapy [19]. The overall 3-year local$\mathrm{PF}$, regional-PF, DM-PF, and overall survival rates were $93.6 \%, 93.3 \%, 86.6 \%$, and $87.2 \%$, respectively. A study in Japan demonstrated the first experience of HT plus chemotherapy for 20 patients with a limited observation period. However, 18 patients who underwent chemotherapy with NDP (cis-diammineglycolatoplatinum, Nedaplatin) and 5FU were in alternating settings. During the alternating chemoradiotherapy and with a median FU of 10.9 months, one patient failed in the regional node and another one failed in the liver. The 10-month OS was $95 \%$ [20]. In the current study, induction chemotherapy and CCRT with HT were well tolerated. During neoadjuvant chemotherapy, only one patient occurred grade 3 leukopenia. No patients experienced grade 3 anemia or thrombocytopenia. Four patients developed grade 3 leukopenia and 1 patient developed grade 3 anemia during the following CCRT with HT treatment. The median treatment time for CCRT was 54 days. The estimated 3-year PF for local, regional, and locoregional survival rates were 92.4\%, 95.7\%, and $88.4 \%$, respectively. HT for locoregionally advanced NPC was shown to be feasible and effective in regard to locoregional control with high compliance, even after neoadjuvant chemotherapy.

Even though nearly $90 \%$ of our patients had locally advanced disease (stage III and IV), patients had excellent locoregional control rates after HT plus 
chemotherapy or even salvage therapy. However, of the 7 relapsed patients in the current study, 4 patients presented distant metastases. The regiment of induction chemotherapy in the current study was CDDP/Epirubicin/5-FU/Leucovoren $(60 / 40 / 2000 / 300 \mathrm{mg} / \mathrm{m} 2)$. Compared to the other studies, the doses of CDDP and Epirubicin in the current study were lower than the other studies $[14,21,22]$. The 3-year DMF survival rate was $78 \%$, suggesting that distant metastases are still the major obstacle to be broken through. Moreover, present regimens of chemotherapy are not effective enough in preventing distant metastases and should be reevaluated.

Higher irradiation doses deliver high rates of locoregional control, progression-free survival for head and neck cancer [23]. However, we may need to be concerned about late complications if the dose is escalated and the volume of tissues are exposed to high doses [24]. On the other hand, if the volume of tissues exposed to high doses is reduced with image-guided IMRT, there is a possibility that treatment could achieve higher locoregional control rate and the probability of such complications could be reduced simultaneously. In the current study, the locoregional failure of 3 patients all belonged to in-field failure. The D95 in PTV70 ranged from 68.8 Gy to 72 Gy and the V97 in PTV70 ranged from $97.3 \%$ to $100 \%$, respectively. (Table 2) None of the out-field or marginal failures noted in the current study showed $3 \mathrm{~mm}$ of PTV's margin, confirming the potential benefits of treating NPC patients with imageguided radiation modality. This finding also suggests that using $3 \mathrm{~mm}$ as the PTV margin in image-guided radiation therapy settings is feasible. Additionally, limited grade 3 of xerostomia, dysphagia and dermatitis were noted in the current setting (Table 6). Moreover, most of patients are normal to grade 1 of late toxicities. Only 4/28 patient had grade 2 xerostomia and 1/28 had grade 2 hearing loss. With accurate image-guided modality, dose escalation with reduced increase of toxicity for OARs becomes more reliable, providing relief for locoregionally advanced NPC patients.

One patient died of cardiac dysfunction, and the possibility that the toxicity was related to epirubicin used in induction chemotherapy should be considered. The range of total dose for epirubicin that cause cardiac toxicities is around $560-600 \mathrm{mg} / \mathrm{m}^{2}[11,25]$. Bonneterre $\mathrm{J}$, et al [25] reported that there were $2 / 85$ cases of congestive heart failure observed after adjuvant treatment with six cycles of fluorouracil $500 \mathrm{mg} / \mathrm{m}^{2}$, epirubicin $100 \mathrm{mg} /$ $\mathrm{m}^{2}$, and cyclophosphamide $500 \mathrm{mg} / \mathrm{m}^{2}$ for breast cancer patients in the 8 years follow up. Hasbini A, et al [11] used mitomycin, 5-fluorouracil, epirubicin, and cisplatin to treat recurrent and metastatic undifferentiated carcinoma of nasopharyngeal and one 42-year-old patient died of cardiac failure which was probably related to the anthracycline-chemotherapy. In the current study, three cycles of $40 \mathrm{mg} / \mathrm{m}^{2}$ induction epirubicin was prescribed and the total dose was $120 \mathrm{mg} / \mathrm{m}^{2}$. Although, the total dose of epirubicin is far from the doses that cause cardiac toxicity.

Even though this innovative approach acquired favorable outcomes with impressive locoregional control and survival result, several limitations need to be addressed. First, our study was retrospective and was carried with inherent biases usual to such a study design. Second, our sample size was small. Thus, these findings should be considered as preliminary and in need of validation in a larger patient group. Third, the study lacked in-house comparable results such as tomotherapy versus conventional IMRT or current regimen versus concurrent chemoradiation. Furthermore, the observation of long-term toxicities should be reported in the future. The clinical benefit of modern IGRT using tomotherapy, hence, could not be fully determined. Due to these limitations, this combination protocol must not be used in the daily practice of treatment for locally advanced NPC.

\section{Conclusions}

In conclusion, this is the first report providing evidence that HT for locoregionally advanced NPC is feasible and effective in regard to locoregional control with high compliance, even after neoadjuvant chemotherapy. No out-field or marginal failure was noted in the current study, confirming the potential benefits of treating NPC patients with image-guided radiation modality. A longterm follow-up study is needed to confirm these preliminary findings.

\section{Author details}

'Division of Radiation Oncology, Department of Radiology, Far Eastern Memorial Hospital, Taipei, Taiwan. ${ }^{2}$ Department of Otolaryngology, Far Eastern Memorial Hospital, Taipei, Taiwan. ${ }^{3}$ Division of Medical Oncology and Hematology, Department of Internal Medicine, Far Eastern Memorial Hospital, Taipei, Taiwan. ${ }^{4}$ Departments of Radiation Oncology, Tri-Service General Hospital, National Defense Medical Center, Taipei, Taiwan. ${ }^{5}$ Institute of Traditional Medicine, School of Medicine, National Yang-Ming University, Taipei, Taiwan.

\section{Authors' contributions}

PWS and BJS drafted the manuscript. LJW, CHH, LJL, PWC, WCL, YMJ and YCL participated in taking care of patients. CHH and PWS carried out all CT evaluations, study design, target delineations and interpretation of the study. $\mathrm{CHH}$ conceived of the study, and participated in its design and coordination. All authors read and approved the final manuscript.

\section{Competing interests}

We have no personal or financial conflict of interest and have not entered into any agreement that could interfere with our access to the data in the research, or upon our ability to analyze the data independently, to prepare manuscripts, and to publish them.

Received: 5 March 2011 Accepted: 13 August 2011

Published: 13 August 2011 


\section{References}

1. Baujat B, Audry H, Bourhis J, Chan AT, Onat H, Chua DT, Kwong DL, AlSarraf M, Chi KH, Hareyama M, Leung SF, Thephamongkhol K, Pignon JP: Chemotherapy in locally advanced nasopharyngeal carcinoma: an individual patient data meta-analysis of eight randomized trials and 1753 patients. Int J Radiat Oncol Biol Phys 2006, 64:47-56.

2. Langendijk JA, Leemans CR, Buter J, Berkhof J, Slotman BJ: The additional value of chemotherapy to radiotherapy in locally advanced nasopharyngeal carcinoma: a meta-analysis of the published literature. $J$ Clin Oncol 2004, 22:4604-12.

3. Huncharek M, Kupelnick B: Combined chemoradiation versus radiation therapy alone in locally advanced nasopharyngeal carcinoma: results of a meta-analysis of 1,528 patients from six randomized trials. Am J Clin Oncol 2002, 25:219-23.

4. Lee NY, Le QT: New developments in radiation therapy for head and neck cancer: intensity-modulated radiation therapy and hypoxia targeting. Semin Oncol 2008, 35:236-50.

5. Mavroidis P, Shi C, Plataniotis GA, Delichas MG, Costa Ferreira B, Rodriguez S, Lind BK, Papanikolaou : Comparison of the helical tomotherapy against the multileaf collimator-based intensity-modulated radiotherapy and $3 \mathrm{D}$-conformal radiation modalities in lung cancer radiotherapy. $\mathrm{Br} J$ Radiol

6. Hsieh CH, Kuo YS, Liao LJ, Hu KY, Lin SC, Wu LJ, Lin YC, Chen YJ, Wang LY, Hsieh YP, Lin SL, Chen CY, Chen CA, Shueng PW: Image-guided intensity modulated radiotherapy with helical tomotherapy for postoperative treatment of high-risk oral cavity cancer. BMC Cancer 2011, 11:37.

7. Hsieh CH, Liu CY, Shueng PW, Chong NS, Chen CJ, Chen MJ, Lin CC, Wang TE, Lin SC, Tai HC, Tien HJ, Chen KH, Wang LY, Hsieh YP, Huang DY, Chen YJ: Comparison of coplanar and noncoplanar intensity-modulated radiation therapy and helical tomotherapy for hepatocellular carcinoma. Radiat Oncol 5:40.

8. Wu WC, Mui WL, Fung WK: Helical tomotherapy of nasopharyngeal carcinoma-any advantages over conventional intensity-modulated radiotherapy? Med Dosim 35:122-7.

9. Trotti A, Colevas AD, Setser A, Rusch V, Jaques D, Budach V, Langer C, Murphy B, Cumberlin R, Coleman CN, Rubin P: CTCAE v3.0: development of a comprehensive grading system for the adverse effects of cancer treatment. Semin Radiat Oncol 2003, 13:176-81.

10. Dinse GE, Lagakos SW: Nonparametric estimation of lifetime and disease onset distributions from incomplete observations. Biometrics 1982, 38:921-32.

11. Hasbini A, Mahjoubi R, Fandi A, Chouaki N, Taamma A, Lianes P, CortesFunes H, Alonso S, Armand JP, Cvitkovic E, Raymond E: Phase II trial combining mitomycin with 5-fluorouracil, epirubicin, and cisplatin in recurrent and metastatic undifferentiated carcinoma of nasopharyngeal type. Ann Oncol 1999, 10:421-5.

12. Lee N, Xia P, Quivey JM, Sultanem K, Poon I, Akazawa C, Akazawa P, Weinberg $V$, Fu KK: Intensity-modulated radiotherapy in the treatment of nasopharyngeal carcinoma: an update of the UCSF experience. Int J Radiat Oncol Biol Phys 2002, 53:12-22.

13. Kwong DL, Pow EH, Sham JS, McMillan AS, Leung LH, Leung WK, Chua DT, Cheng AC, Wu PM, Au GK: Intensity-modulated radiotherapy for earlystage nasopharyngeal carcinoma: a prospective study on disease control and preservation of salivary function. Cancer 2004, 101:1584-93.

14. Kam MK, Teo PM, Chau RM, Cheung KY, Choi PH, Kwan WH, Leung SF, Zee B, Chan AT: Treatment of nasopharyngeal carcinoma with intensitymodulated radiotherapy: the Hong Kong experience. Int J Radiat Oncol Biol Phys 2004, 60:1440-50.

15. Wolden SL, Chen WC, Pfister DG, Kraus DH, Berry SL, Zelefsky MJ: Intensitymodulated radiation therapy (IMRT) for nasopharynx cancer: update of the Memorial Sloan-Kettering experience. Int J Radiat Oncol Biol Phys 2006, 64:57-62.

16. Lin JC, Jan JS, Hsu CY, Liang WM, Jiang RS, Wang WY: Phase III study of concurrent chemoradiotherapy versus radiotherapy alone for advanced nasopharyngeal carcinoma: positive effect on overall and progressionfree survival. J Clin Oncol 2003, 21:631-7.

17. Lee N, Harris J, Garden AS, Straube W, Glisson B, Xia P, Bosch W, Morrison WH, Quivey J, Thorstad W, Jones C, Ang KK: Intensity-modulated radiation therapy with or without chemotherapy for nasopharyngeal carcinoma: radiation therapy oncology group phase II trial 0225. J Clin Oncol 2009, 27:3684-90.
18. Kim K, Wu HG, Kim HJ, Sung MW, Kim KH, Lee SH, Heo DS, Park Cl: Intensity-modulated radiation therapy with simultaneous integrated boost technique following neoadjuvant chemotherapy for locoregionally advanced nasopharyngeal carcinoma. Head Neck 2009, 31:1121-8.

19. Wong FC, Ng AW, Lee VH, Lui CM, Yuen KK, Sze WK, Leung TW, Tung SY: Whole-field simultaneous integrated-boost intensity-modulated radiotherapy for patients with nasopharyngeal carcinoma. Int J Radiat Oncol Biol Phys 76:138-45.

20. Kodaira T, Tomita N, Tachibana H, Nakamura T, Nakahara R, Inokuchi H, Fuwa N: Aichi cancer center initial experience of intensity modulated radiation therapy for nasopharyngeal cancer using helical tomotherapy. Int J Radiat Oncol Biol Phys 2009, 73:1129-34

21. Ma J, Mai HQ, Hong MH, Min HQ, Mao ZD, Cui NJ, Lu TX, Mo HY: Results of a prospective randomized trial comparing neoadjuvant chemotherapy plus radiotherapy with radiotherapy alone in patients with locoregionally advanced nasopharyngeal carcinoma. J Clin Oncol 2001, 19:1350-7.

22. Al-Amro A, Al-Rajhi N, Khafaga Y, Memon M, Al-Hebshi A, El-Enbabi A, ElHusseiny G, Radawi A, Belal A, Allam A, El-Sebaie M: Neoadjuvant chemotherapy followed by concurrent chemo-radiation therapy in locally advanced nasopharyngeal carcinoma. Int J Radiat Oncol Biol Phys 2005, 62:508-13.

23. Miah AB, Bhide SA, Guerrero-Urbano MT, Clark C, Bidmead AM, St Rose $S$, Barbachano Y, A'Hern R, Tanay M, Hickey J, Nicol R, Newbold KL, Harrington KJ, Nutting CM: Dose-Escalated Intensity-Modulated Radiotherapy Is Feasible and May Improve Locoregional Control and Laryngeal Preservation in Laryngo-hypopharyngeal Cancers. Int J Radiat Oncol Biol Phys

24. Mohan R, Wu Q, Manning M, Schmidt-Ullrich R: Radiobiological considerations in the design of fractionation strategies for intensitymodulated radiation therapy of head and neck cancers. Int J Radiat Oncol Biol Phys 2000, 46:619-30.

25. Bonneterre J, Roche $H$, Kerbrat $P$, Fumoleau $P$, Goudier MJ, Fargeot $P$, Montcuquet P, Clavere P, Barats JC, Monnier A, Veyret C, Datchary J, Van Praagh I, Chapelle-Marcillac I: Long-term cardiac follow-up in relapse-free patients after six courses of fluorouracil, epirubicin, and cyclophosphamide, with either 50 or $100 \mathrm{mg}$ of epirubicin, as adjuvant therapy for node-positive breast cancer: French adjuvant study group. $J$ Clin Oncol 2004, 22:3070-9.

doi:10.1186/1748-717X-6-95

Cite this article as: Shueng et al.: Concurrent image-guided intensity modulated radiotherapy and chemotherapy following neoadjuvant chemotherapy for locally advanced nasopharyngeal carcinoma. Radiation Oncology 2011 6:95.

\section{Submit your next manuscript to BioMed Central and take full advantage of:}

- Convenient online submission

- Thorough peer review

- No space constraints or color figure charges

- Immediate publication on acceptance

- Inclusion in PubMed, CAS, Scopus and Google Scholar

- Research which is freely available for redistribution

Submit your manuscript at www.biomedcentral.com/submit
C Biomed Central 